\title{
3D printed "Starmix" drug loaded dosage forms for paediatric applications
}

\author{
Nicolaos Scoutaris ${ }^{1}$, Steven A. Ross ${ }^{1}$, Dennis Douroumis ${ }^{1 *}$ \\ ${ }^{1}$ Faculty of Engineering and Science, School of Science, University of Greenwich, Chatham \\ Maritime, Chatham, Kent ME4 4TB, UK
}

\footnotetext{
* Correspondence: Faculty of Engineering and Science, University of Greenwich, Medway Campus, Chatham Maritime, Kent ME4 4TB, UK. Tel.: +44 (0) 208331 8440; Fax: +44 (0) 2083319805.

E-mail address: D.Douroumis@gre.ac.uk
} 


\section{Abbreviations List}

HME

Hot melt extrusion processing

IND

Indomethacin

HPMCAS

Hypromellose acetate succinate

PEG

DSC

XRPD

FT-IR

SEM

HPLC

TGA

(3D) printing has received significant attention as a manufacturing process for pharmaceutical dosage forms. In this study, we used Fusion Deposition Modelling (FDM) in order to print "candy - like" formulations by imitating Methods Hot melt extrusion processing (HME) was coupled with FDM to prepare extruded filaments of indomethacin (IND), hypromellose acetate succinate (HPMCAS) and olyethylene glycol (PEG) formulations and subsequently feed them in the 3D printer. The shapes of the Starmix ${ }^{\circledR}$ objects were printed in the form of a heart, ring, bottle, ring, bear and . Differential scanning calorimetry (DSC), X-ray powder diffraction (XRPD), Fourier

Keywords: 3D printing, paediatric medicines, fusion deposition modelling, hot melt extrusion, taste masking

Polyethylene glycol

Differential scanning calorimetry

X-ray powder diffraction

Fourier Transform Infra-red Spectroscopy

Scanning Electron Microscopy

High performance liquid chromatography

Thermogravimetric analysis 


\section{INTRODUCTION}

The development of high quality, effective and safe paediatric medicines has been proven a challenging process for the pharmaceutical industry. Due to the substantial differences in the drug development process for paediatric patients and adult patients, new regulations were introduced by the European Medicines Agency (EMEA) in 2007, which governed the drug production strategies for paediatric medications. According to Strickley et al., (2007) development difficulties are related to dose modification, ease of administration/swallowing, taste masking, physical stability, packaging and providing/designing the measuring device [1].

The oral route of administration is often utilized as the route of choice for the delivery of medical products to paediatric patients, in both solid and liquid forms including; syrups, suspensions and emulsions; powders, granules, effervescent tablets, orodispersible tablets, chewable tablets and gums; mini tablets, innovative granules; and both conventional immediate release and modified release tablets and capsules [2]. A great disadvantage of liquid medications is the higher chance of error when measuring dosage for selfadministration. A number of studies have demonstrated that patients who self administer liquid medications have an estimated error rate of $50 \%$ to $60 \%$, due to their inability to measure the correct dose [3-5]. This problem can be avoided by manufacturing solid oral dosage forms which offer a number advantages over the liquid forms, these include; having a lower cost of development, easy administration, greater stability, dose accuracy, have the capacity to develop modified release formulations, can be produced with greater uniformity and have greater taste masking capabilities [6]. Although oral administration is the most common route for paediatric populations, the development of palatable formulations is complicated. The developed medicines should be easily swallowed, taste masked while maintaining safety, efficacy, accessibility and affordability. Nevertheless, there is an increased demand for the development of novel technologies for paediatric patients that mainly focus on the formulation design and/or administration/dosing devices [7].

Such a novel technology is $3 \mathrm{D}$ printing, which encompasses a wide range of additive manufacture processes, where products are designed using Computer Aided Design (CAD) software [8-11]. The product is then processed by a slicing software that divides the object into thin cross sections. These are then printed out one on top of the other, forming compact layers which form the 3D structure. The primary objective of the various 3D printing technologies is to develop and produce models and prototypes of the designed product in a rapid and inexpensive manner [12]. The epitome of 3D printing applications for medicinal products is the recently approved 3D printed drug product called Spritam (levetiracetam) manufactured by using the Zipdose Technology based on a powder bed-liquid 3D printing technology.

An increasing number of researchers are employing 3D printing technologies to develop oral dosage forms, with modified drug release profiles to allow either sustained or immediate drug release [13-15]. Recently, 3D printing was utilized to create a multi-active solid dosage form, containing 5 different API compartmentalised within the same capsule, which are autonomously controlled with two separate release profiles, called Polypill [16]. An interesting approach was presented by Goyannes et al. where an anti-acne drug (salicylic 
acid) loaded structure in the shape of a nose was prepared by comparing Fused Deposition Modelling (FDM) and stereolithography [17].

However, FDM has proven to be one of the more versatile 3D printing methods due to the range of potential printing materials, which can be utilized on the system. However, further development of FDM extruders have allowed for the printing of almost any thermoplastic material such as polycarbonate and polyurethane [18]. Recently, the utilisation of FDM was extended to pharmaceutical materials where filaments of drugs and polymers are prepared and extruded through an FDM 3D printer. Specifically, FDM has been utilised to prepare formulations of differing shapes and infill ratio's in order to tune the drug release profile $[19,20]$. As expected tablets weights increased with an increasing infill percentage and same size, demonstrating high reproducibility in physical dimensions, along with high mechanical strength which is resistant to damage upon handling. Moreover, extended release tablet of prednisolone were fabricated by using loaded poly(vinyl alcohol) (PVA) filaments [21]. The precision of dose control ranged between $88.7 \%$ and $107 \%$ while prednisolone existed in amorphous form within the PVA matrix and released from the 3D printed tablet over a $24 \mathrm{hr}$ period. Other work involves the development of Patient-Specific Immediate Release Tablets using theophylline loaded filaments as a mixture with PVP in the presence of plasticiser produced by means of hot melt extrusion. A 3D printing technique was applied to produce caplet-shaped tablets with excellent mechanical properties and an immediate in-vitro release pattern [22]. The aforementioned examples demonstrate that by simply modifying the printing patterns and structure, without altering the polymer-drug properties, it is possible to modify the drug dissolution rates.

The aim of this work was to introduce the feasibility of 3D printing coupled with hot melt extrusion to prepare paediatric medicines that can be consumed easily by children from 2 - 11 years old. The medicines were designed in such a way to imitate "sweet - like" chewable tablets. Chewable tablets are a valuable paediatric dosage form and have been used for various products such as antacids, antibiotics, anticonvulsants, analgesics, antiasthmatics and cold/allergy preparations. The proposed 3D printing approach improves compliance and adherence of children to the prescribed medication. In addition the combined HME and 3D printing benefits such as taste masking and easy to swallow respectively, lead to dose accuracy and enhanced palatability. For the purposes of the study "Starmix $®$ " (HARIBO PLC, UK) designs were printed using IND as model drug substance and HMPCAS as the polymeric excipient. Indomethacin is a nonsteroidal anti-inflammatory drug commonly used as a prescription medication to reduce fever, pain, stiffness, and swelling from inflammation and HPMCAS is a thermoplastic polymer which has been used primarily as an enteric coating material and dissolves at $\mathrm{pH} 5.5-6.8$.

\section{MATERIALS AND METHODS}

\section{Materials}

Indomethacin (IND) and polyethylene glycol (PEG) were purchased from Tokyo Chemicals (Belgium) and Sigma - Aldrich (U.K.) respectively. Hypromellose acetate succinate (HPMCAS, AQOAT AS-MF) was kindly donated by Shin - Etsu (Japan). 


\section{Extrusion of IND - HPMCAS filament}

A physical blend composed of indomethacin, HPMCAS and PEG 6000 at 20/60/20 $\mathrm{wt} / \mathrm{wt}$ ratio were accurately weighed and homogeneously blended in a Turbula shaker-mixer at 100rpm for 10 minutes. The blended physical mixer was loaded into a DD Flexwall 18 feeder (Brabender Technology, Germany), and fed into a twin-screw extruder (Eurolab 16, Thermo Fisher, Germany) at a rate of $10 \mathrm{~g}$ per minute. A circular shaped die was employed to yield polymer strand extrudates. The 9 heating zones across the barrel were set at the following temperature profile; $40^{\circ} \mathrm{C}, 55^{\circ} \mathrm{C}, 75^{\circ} \mathrm{C}, 100^{\circ} \mathrm{C}, 140^{\circ} \mathrm{C}, 140^{\circ} \mathrm{C}, 140^{\circ} \mathrm{C}, 140^{\circ} \mathrm{C}, 120^{\circ} \mathrm{C}$ with the die temperature set at $80^{\circ} \mathrm{C}$. The barrel was slightly cooled towards the end to allow the mixture to re-solidify, ensuring the end-product was extruded as a strand, which is uniform in diameter. A screw speed of 50 rpm was utilised.

\section{D printing process of paediatric formulations}

The Starmix ${ }^{\circledR}$ structures were printed by using a standard FDM printer HD2xR Airwolf (U.S.A). The structures were designed with SolidWorks (Dassault Systems, USA) and exported as .stl files which were subsequently imported into Cura software to generate the Gcode for the printing. The print temperature was $165^{\circ} \mathrm{C}$ and the print speed was set at $25 \mathrm{~mm} / \mathrm{sec}$. The infill percentage was $7 \%$ and the layer height $150 \mu \mathrm{m}$. The size of the perimeter shell was $0.5 \mathrm{~mm}$ and the top and bottom shell $0.4 \mathrm{~mm}$. No supports or rafts were utilised in the printed model.

\section{Scanning Electron Microscopy (SEM)}

The morphology of the extruded polymer strands and the 3D printed structure was observed via scanning electron microscopy (Hitachi SU8030, Japan). Both Samples were placed on an aluminium stub and coated with a thin layer of Mikrostik nonconductive adhesive (Agar scientific, UK). The samples were then plated with chromium under an atmosphere of argon. SEM images were taken using an electron beam accelerating voltage of $2 \mathrm{kV}$. Images were captured between $\times 30$ and 2.00k magnifications.

\section{X-Ray Powder Diffraction (XRPD)}

X-ray powder diffraction was used to determine the solid state of the bulk materials, the extruded polymer strand and the polymer strand after 3D Printing. This was done using a D8 advanced x-ray Diffractometer (Bruker, Germany) in reflection mode. A Cu anode X-ray tube was powered at $40 \mathrm{kV}$ and $40 \mathrm{~mA}$. A primary Göbel mirror was used for the parallel beam and the removal of $\mathrm{CuK} \beta$. A primary $4^{\circ}$ Soller slit, a secondary $2.5^{\circ}$ Soller slit and a $0.6 \mathrm{~mm}$ exit slit was selected for this experiment. Each sample was scanned from 2 to $60^{\circ} 2 \theta$ with a step size of $0.02^{\circ} 2 \theta$ degrees/step and a counting time of $0.2 \mathrm{sec} / \mathrm{step}$. As the polymer samples were difficult to crush to a small enough size to run for XRPD, they were first doused in liquid nitrogen then ground in a mortar and pedestal, before being placed in a low background holder and inserted into the device. DIFFRAC.EVA software was used for the phase analysis.

\section{Differential Scanning Calorimetry (DSC)}

The temperature profiles of the bulk ingredients and extruded strands were analysed using a differential scanning calorimeter (Mettler-Toledo 823e, Greifensee, Switzerland). The 
samples were accurately weighed between $3 \mathrm{mg}-5 \mathrm{mg}$ and placed into an aluminium pan and crimped. Each sample was heated from $0^{\circ} \mathrm{C}$ to $300^{\circ} \mathrm{C}$ at $10^{\circ} \mathrm{C} / \mathrm{min}$ heating rate except for PEG 6000 which was heated to $150^{\circ} \mathrm{C}$. Nitrogen gas was used as a purge, at a flow rate was set at $50 \mathrm{ml} / \mathrm{min}$. STARe excellence software was used to analyse the data.

\section{Fourier Transform Infra-red Spectroscopy (FT-IR)}

FTIR analysis was performed on the drug, carrier, drug/carrier physical mixtures, and complex using PerkinElmer PE1600 (Massachusetts 02451, USA) Fourier transform infrared spectra according to the $\mathrm{KBr}$ disc method from 400 to 3600 wavelength/cm-1 range. For FTIR analysis, amorphous IND was prepared by using solvent evaporation method where IND dissolved in methanol following by rapid evaporation of the solvent.

\section{Confocal Raman analysis}

Room temperature Raman spectra of the "pure" formulation components were obtained using a Jobin/Yvon LabRam 320 instrument equipped with an Olympus microscope (Horiba, Japan). The spectrometer is equipped with an 1800 grooves $/ \mathrm{mm}$ holographic grating, a holographic notch filter, a Peltier-cooled CCD (MPP1 chip) for detection, and an Olympus BX40 microscope. An Ar+ ion laser $(\lambda=633 \mathrm{~nm})$ was used. Raman spectra of solid-state samples were collected at room temperature on a microscope slide using a microscope objective of $50 \times$ magnification to focus the laser beam. A backscattering $\left(180^{\circ}\right.$ between excitation and collection) geometry was used in all experiments. Each spectral scan was collected for $5 \mathrm{~s}$ using 4 accumulations. The Raman instrument was calibrated using the v1 line of silicon at $520.7 \mathrm{~cm}^{-1}$.

Raman mapping was performed using a Jobin/Yvon LabRam 320 instrument equipped with an Olympus microscope (Horiba, Japan) by means of Ar+ ion laser $(\lambda=532.8$ $\mathrm{nm}$ ) and $1800 \mathrm{l} / \mathrm{nm}$ grating on 3 different particles for each formulation. The experimental conditions were: $100 \mathrm{~nm}$ slit width, a 50x Microsoft objective and $0.4 \mathrm{~s}$ acquisition time. Each spectrum was the mean of the two. The sample profiling was performed at step increments of $3 \mu \mathrm{m}$ in the $\mathrm{X}-\mathrm{Y}$ direction covering the biggest possible surface of the part. Principal component analysis (PCA) was applied to identify the number of components and plot the chemical maps. Prior to analysis, the spectra baseline was checked by using the automatic weighted least square and normalised to the unit area to avoid deviation among the Raman spectra.

\section{Taste masking of 3D designs}

In vivo taste masking evaluation was performed on 10 healthy human volunteers from whom informed consent was first obtained (approved by the Ethics Committee of the University of Greenwich). The study is also in accordance to the Code of Ethics of the World Medical Association (Declaration of Helsinki). The healthy volunteers of either sex (age 1825) were selected, trained and the one Stramix design was held in the mouth for another 120 $\mathrm{s}$, and then spat out. The mouth was rinsed with water without swallowing the tablet. The equivalent of $50 \mathrm{mg}$ of bulk IND was held in the mouth for $30 \mathrm{~s}$ and then spat out. Bitterness was recorded immediately to the bitterness intensity scale from 1 to 5 where $1,2,3,4$, and 5 indicate no, threshold, slight, moderate, and strong bitterness. 


\section{In vitro dissolution studies}

Dissolution studies were conducted by using a USP II paddle apparatus (Varian 705, US). The amount of extruded powders was equivalent to $25 \mathrm{mg}$ of IND, placed into $900 \mathrm{~mL}$ of phosphate buffer solution ( $\mathrm{pH}$ 6.8), in each dissolution vessel $(\mathrm{n}=3)$. The temperature of the dissolution media was maintained at $37{ }^{\circ} \mathrm{C}$ with a paddle rotation of $100 \mathrm{rpm}$. Samples, about 2-3 mL, were withdrawn at 10, 20, 30, 60, 90 and 120 min intervals and filtered with a $200 \mu \mathrm{m}$ filter prior to HPLC analysis.

\section{HPLC analysis}

The content of IND in dissolution samples was determined by HPLC. The samples were analysed on an HPLC system (Agilent Technologies, 1200 series) equipped with a quaternary pump using a Hichrom S50DS2-4889 $(5 \mu \mathrm{m} \times 150 \mathrm{~mm} \times 4 \mathrm{~mm})$ column. The column temperature time was set at $25{ }^{\circ} \mathrm{C}$ while the retention time was $4 \mathrm{~min}$. The mobile phase consisted of 70:30:0.2 (v/v/v) methanol/water/acetic acid and the injected sample volumes were $20 \mu \mathrm{L}$, the flow rate was $1.5 \mathrm{~mL} / \mathrm{min}$, and the detector was set at a wavelength of $260 \mathrm{~nm}$. The calibration curve for IND was plotted over a concentration range of 10-50 $\mu \mathrm{g} / \mathrm{mL}$.

\section{RESULTS AND DISCUSSION}

\section{Hot melt extrusion (HME) and 3D printing coupling}

HME is a well-known processing pharmaceutical technology that has been successfully used over the past twenty years to increase the solubility of water insoluble drugs and mask the taste of bitter APIs for paediatric applications by producing solid dispersions [23]. In this study, HME was employed as the core technique to produce printing filaments and subsequently coupled with a 3D printer. HME nowadays is the predominant technique to create filaments of pharmaceutical compounds which can be subsequently inserted into the 3D printer. The active pharmaceutical ingredient (API) is introduced either by soaking [24] or by extrusion [25-27]. IND was selected as a model API as it has been previously reported to produce extruded solid dispersions with increased solubility while HPMCAS is a suitable thermoplastic career for manufacturing of drug loaded strands. The main aim of the study was to manufacture taste masked IND/HPMCAS solid dispersions with increase drug solubility in the form of strands/filaments that could be fed to the 3D printer and produce paediatric chewable tablets. According to Lopez et al., the capability of children to swallow solid dosage forms is a major issue and thus chewable tablets are an excellent paediatric dosage form [3]. They can be administrated to children of 2 years old and older where swallowing or disintegration is assisted by the patient.

The design of the chewable tablets was inspired by the Starmix flavour gummy sweets (HARIBO plc.) to appear as "candy - like" formulations. By imitating these popular confectionaries the 3D printed chewable tablets aim to improve patient compliance and palatability. 


\section{Process optimisation}

Premixed drug, polymer and plasticiser blends were fed into the extruder in order to produce drug-loaded filaments suitable for 3D printing. One of the most critical features is the filament quality, which should be flexible enough to prevent any breakage printing. For that purpose, PEG was added as a plasticiser to facilitate better extrusion processing and enhance the strand flexibility. Various polymer/plasticiser ratios were tested (data not shown) to concluded that $20 \%$ PEG was giving the best filament quality. The diameter of the filament was adjusted between 2.7 and $2.9 \mathrm{~mm}$, to meet the $3 \mathrm{D}$ printer specifications. Due to the HPMCAS/PEG swelling at the end of the extrusion process a die of $1 \mathrm{~mm}$ was selected to process the formulations.

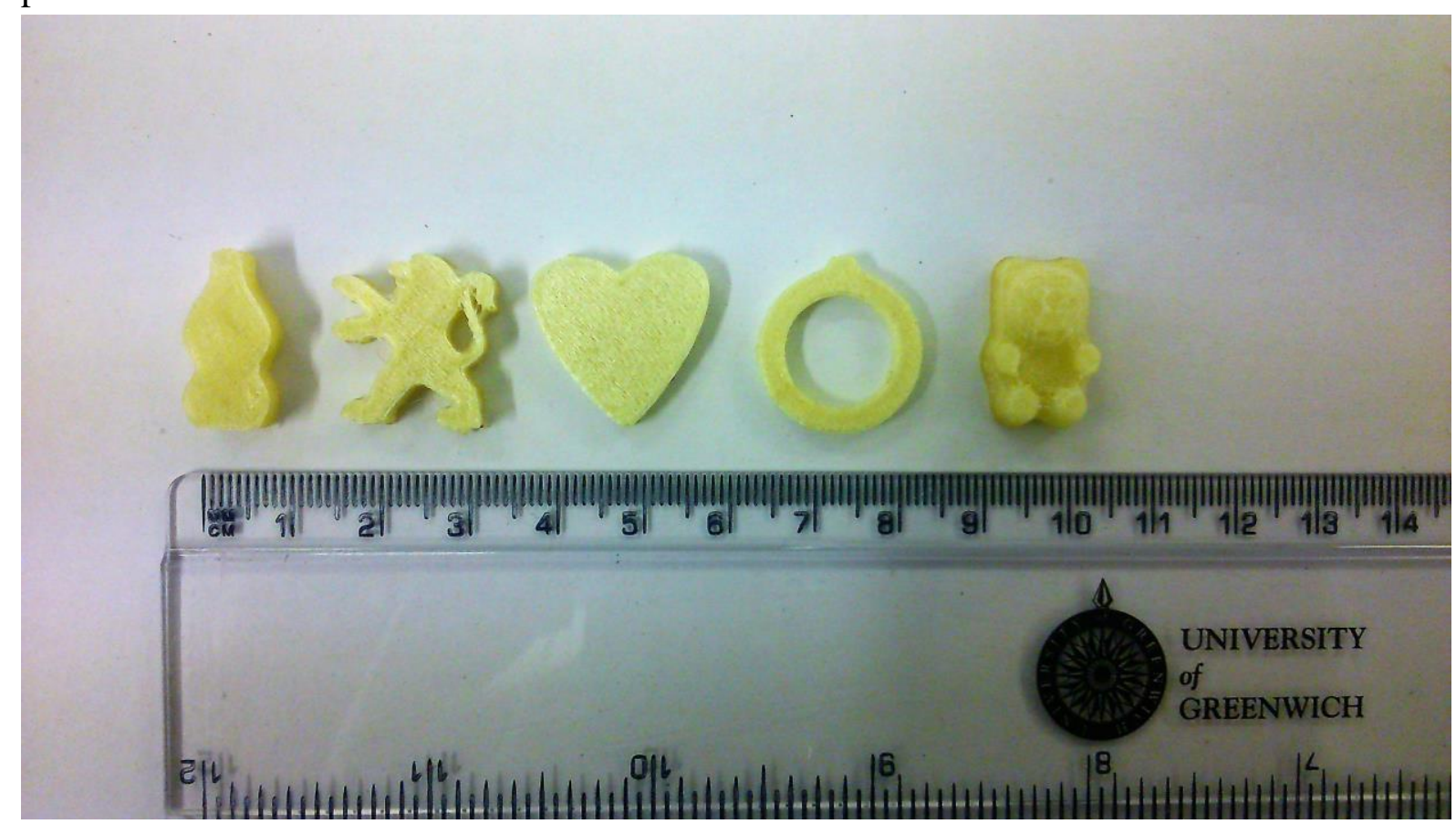

Fig. 1: Photographic image of the 3D printed medicines loaded with IND as model substance

As shown in Fig. 1 various Starmix $®$ IND loaded structures were printed at a wide range of designs. By using SolidWorks the process was programmed with $5 \%$ infill ratio $25 \mathrm{~mm} \mathrm{~s}^{-1}$ printing speed and $160 \mu \mathrm{m}$ layer thickness in order to print doses of $25 \mathrm{mg}$ IND per structure. The printing time for each Starmix $®$ tablet was $5 \mathrm{~min}$.

The $3 \mathrm{D}$ printing optimisation process was a prerequisite to achieving excellent bonding between the layers and thus fine $3 \mathrm{D}$ printed structures. All Starmix $®$ structures were printed at temperatures of $165^{\circ} \mathrm{C}$ much higher than those used to extrude the exact same formulations. This can be explained by the effect of the extrusion process (e.g. screw configuration, temperature profile), which results in higher torque and consequently reduces significantly the melt viscosity of the extruded formulations. In addition, due to the small size of the $3 \mathrm{D}$ printing nozzle further temperature increase is required to reduce the melt viscosity of the filament and achieve the optimum printing conditions. Due to the complexity of the designs the weight of the individual printed forms was adjusted to deliver $50 \mathrm{mg}$ of IND according to the initial drug/polymer ratio. The 3D printing of Starmix $®$ designs was 
highly accurate, reproducible and produced smooth structures without any defects. inconsistencies of the filament thickness. The variations observed on tablet size were negligible.

\section{Scanning Electron Microscopy}

The topography of the filament and the 3D printed structure was investigated by means of SEM. As illustrated in Fig 2a, the hot melt extruded filament presented a homogeneous and very smooth surface without any defects suggesting a perfect extrusion processing. No drug particles could be observed on the filament surface indicating very good IND blending and content uniformity (see further analysis). The manufacturing of high quality filaments is a prerequisite in order to facilitate robust $3 \mathrm{D}$ printing process with the required resolution of the printed designs. Fig. $2 \mathrm{c}$ shows the layer by layer deposition of the printed layers which appear to be uniform with close packing. The thickness of the layers were estimated at $150-180 \mu \mathrm{m}$.

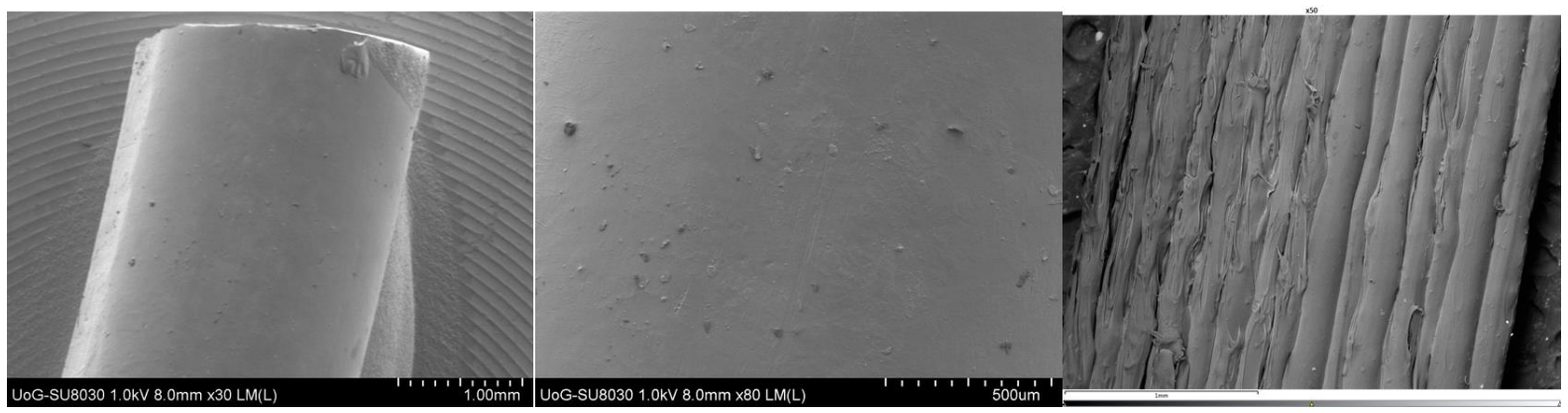

Fig. 2: SEM images of a) extruded filament, b) surface of the extruded filament and c) surface of $3 D$ structure

\section{Thermal Analysis}

Thermogravimetric analysis (TGA) was used to examine the thermal stability of pharmaceutical ingredients under the hot melt extrusion and 3D printing processing temperatures. In Fig. 3, IND showed that a negligible mass loss $(0.5 \%)$ up to $170^{\circ} \mathrm{C}$ followed by a fast mass loss at around $220^{\circ} \mathrm{C}$ due to its degradation. AQOAT exhibited a small mass loss $(2.7 \%)$ from $30-170^{\circ} \mathrm{C}$ and a second at $250^{\circ} \mathrm{C}$ which corresponds to its degradation. PEG was extremely stable at high temperatures without any losses and only presented a fast mass loss at $320^{\circ} \mathrm{C}$ due to its degradation. Both filament and the $3 \mathrm{D}$ printed Starmix structures demonstrates identical mass loss pathways showing that all mixtures remain stable up to $250^{\circ} \mathrm{C}$ with $2.8 \%$ mass loss, which is higher than the degradation temperature of IND indicating interactions among the polymers and the drug substance. Above this temperature, both samples exhibited a fast mass loss due to degradation. Overall, the TGA profiles demonstrate that the HME filament is suitable for $3 \mathrm{D}$ printing temperature at $165^{\circ} \mathrm{C}$. 


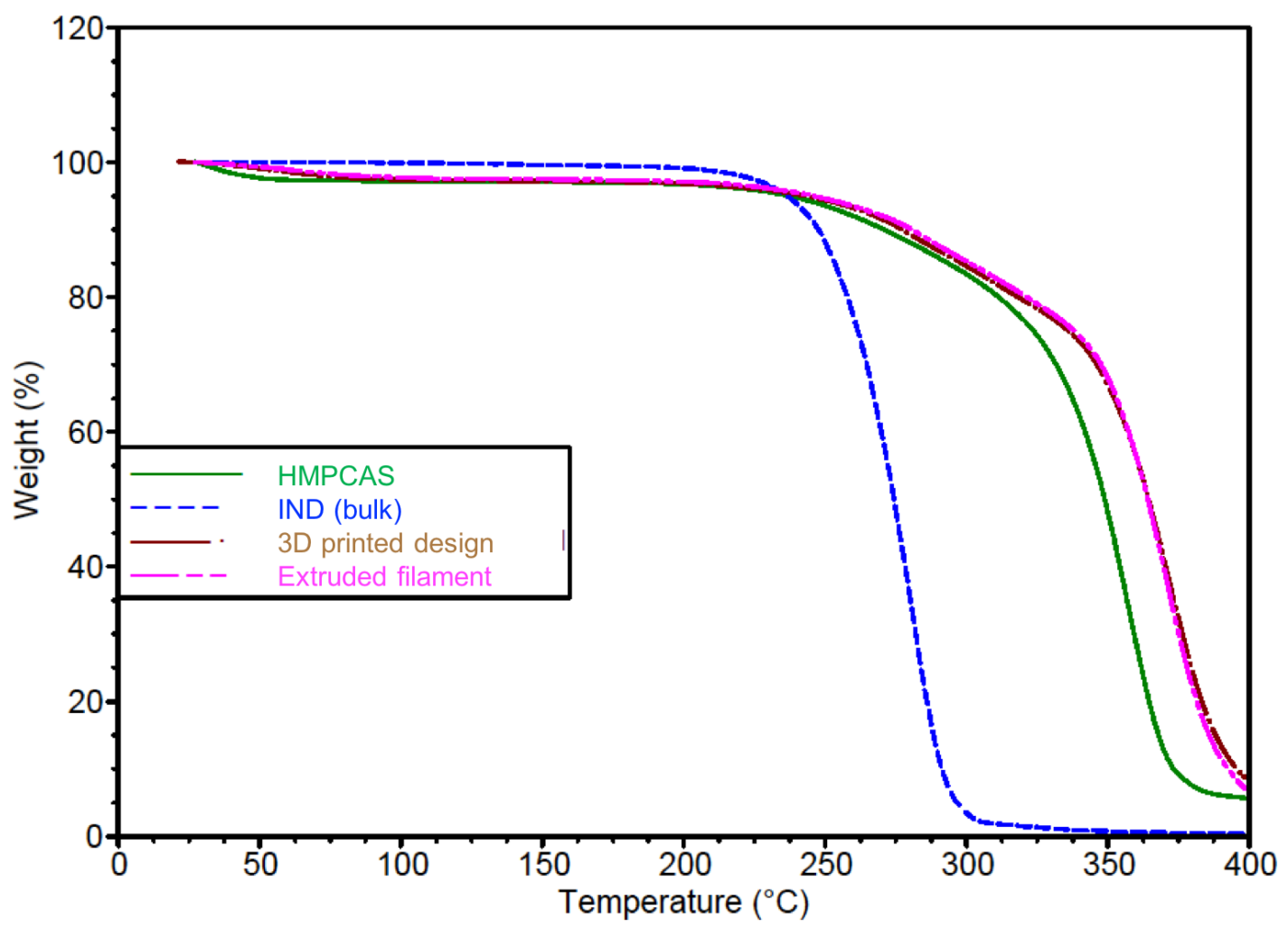

Fig. 3: TGA graph of the bulk IND, HPMCAS, PEG, extruded filament and $3 D$ printed structure

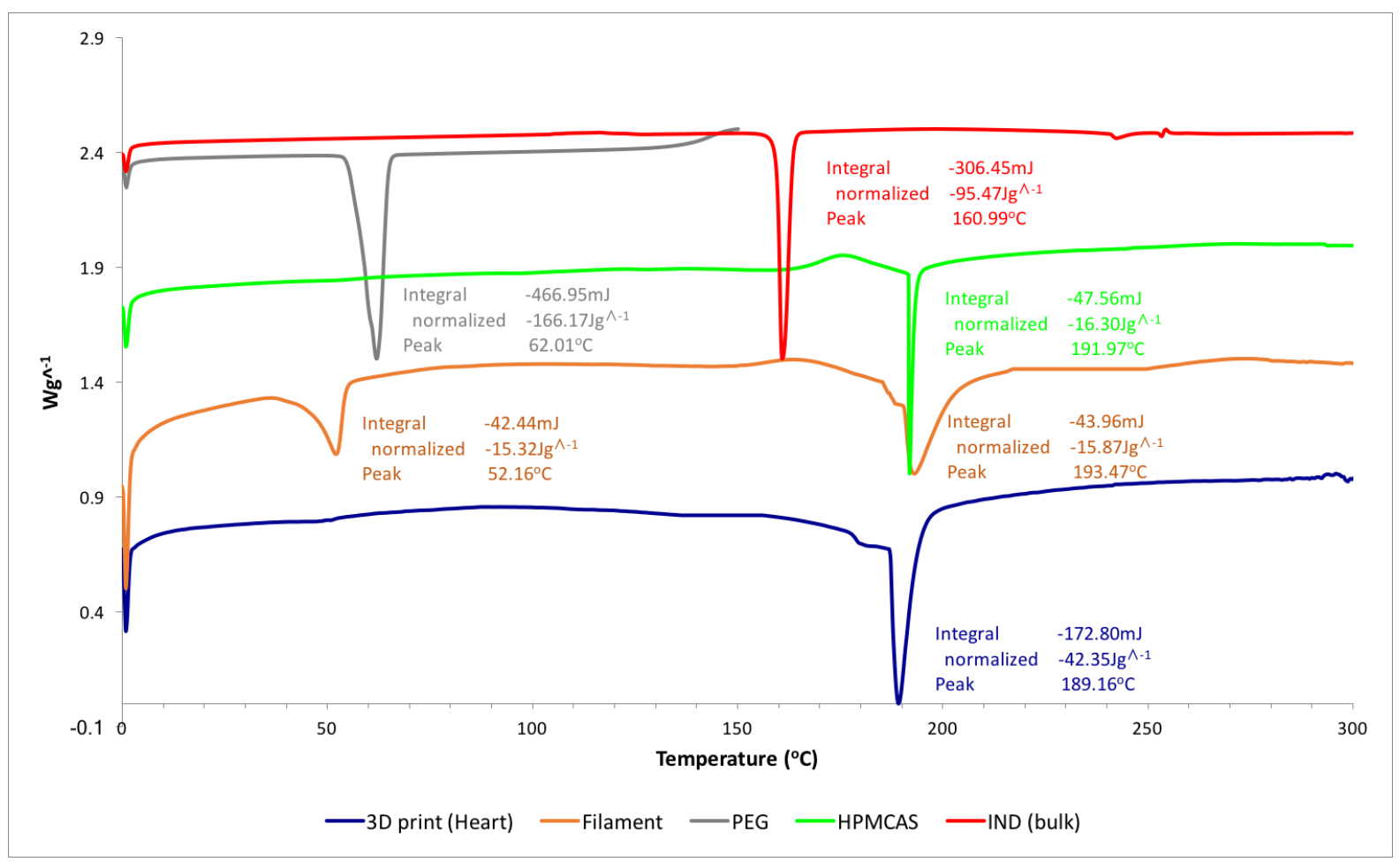

Fig. 4: DSC thermograph of pure IND, PEG, HPMCAS, extruded filament and $3 D$ printed strucutre 
DSC was conducted to investigate the physicochemical properties of the bulk strong endothermic peak at $160.99^{\circ} \mathrm{C}$ which is typical for the crystalline $\gamma$-form while PEG 6000 exhibits a melting endotherm at $62^{\circ} \mathrm{C}$. HPMCAS is an amorphous polymer with a glass transition at $131^{\circ} \mathrm{C}$ while it presents an exothermic peak at $175^{\circ} \mathrm{C}$ indicating crystallisation followed by a degradation endothermic peak at $191.8^{\circ} \mathrm{C}$. The extruded filament shows a melting endotherm at $52.16^{\circ}$ corresponding to PEG 6000 melting point suppression while no IND melting endotherm can be observed suggesting that the drug is in amorphous or molecularly dispersed form. A thermal endotherm appears at $223.5^{\circ} \mathrm{C}$ which is probably related to the HPMCAS degradation. Interestingly, in the thermogram of the $3 \mathrm{D}$ printed structures the PEG 6000 melting endotherm has disappeared suggesting that the polymer has turned to its amorphous state due to the second thermal processing (3D extrusion).

\section{X-Ray Diffraction analysis}

X-ray analysis was performed in order to verify the physical state of IND in the 3D printed designs. The XRD diffractogram of bulk IND, HPMCAS, filament and 3D printed are illustrated in Fig. 5. The XRD of bulk IND presents strong intensity peaks at $11.6^{\circ}, 16.4^{\circ}$, $19.6^{\circ}, 26.89^{\circ}$, and $29.3^{\circ} 2 \theta$ values which correspond to $\gamma$-IND [28]. The HPMCAS diffractogram does not depict any intensity peaks due to the amorphous nature of the polymer, while PEG showed strong intensity peaks at $15^{\circ}$ and $24^{\circ}$. The XRD of the extruded filament revealed two small peaks at $15^{\circ}$ and at $24^{\circ} 2 \theta$ values, which are indicative of PEG suggesting that a part of the plasticiser is still in the crystalline state. This observation is also in good agreement with the DSC analysis where the PEG melting endotherm was identified. In contrast, the diffractograms of the 3D printed structure did not reveal any intensity peaks and the two PEG peaks have disappeared indicating that PEG has turned to completely amorphous state. This was attributed to the effect of the filament re-heating during the printing process, which occurs at higher temperature compared to the HME process.

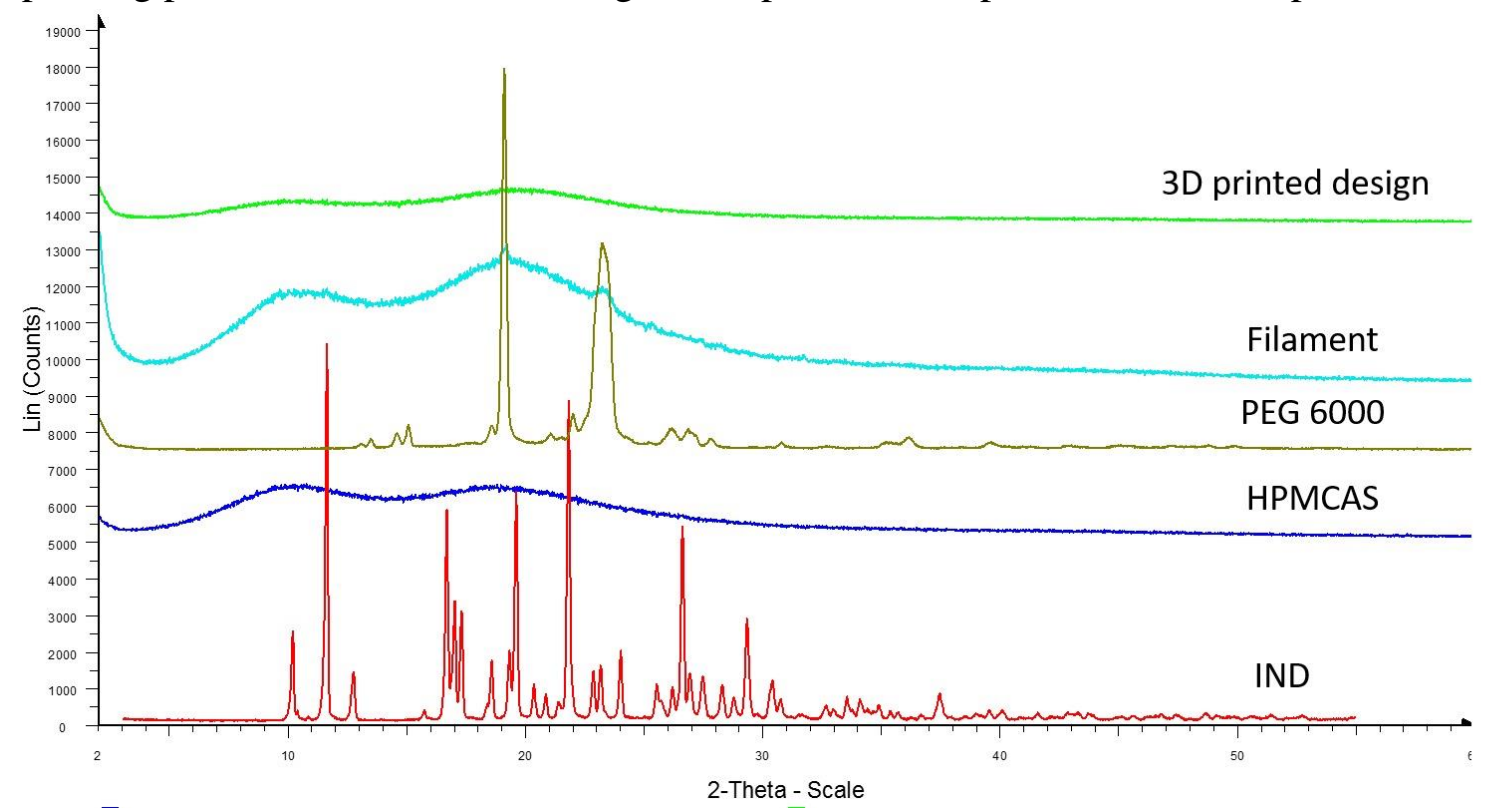

Fig. 5: XRD graphs of IND, HPMCAS, PEG, extruded filament and 3D printed structure 


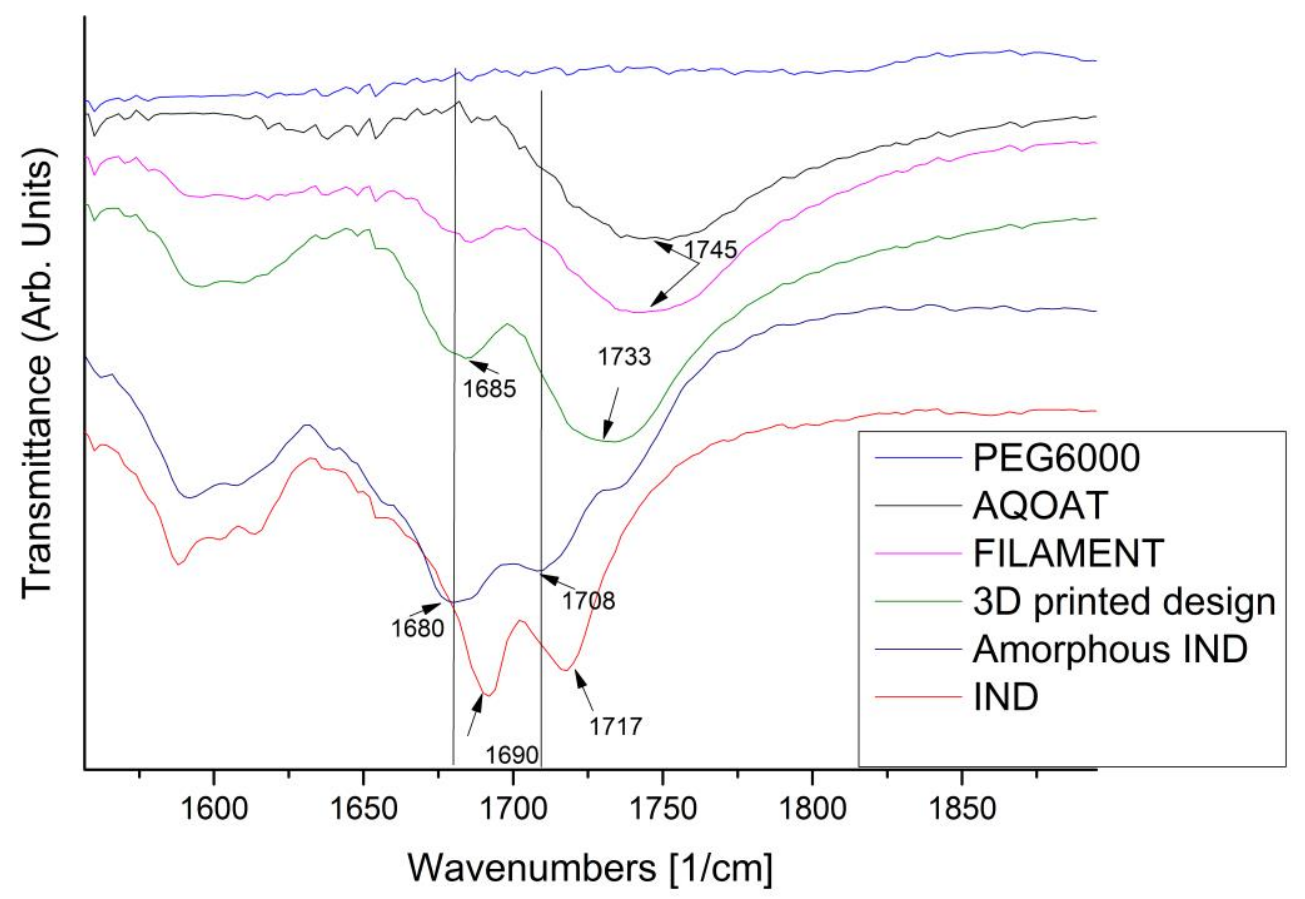

Fig. 6: FT-IR graphs of PEG, HPMCAS, Filament, 3D printed mixture Amorphous IND and powder IND in the region of $1550-1900 \mathrm{~cm}^{-1}$ examine possible interactions among the compounds. Specifically, the carbonyl groups (-CO) and hydroxyl group (-OH) of IND can form hydrogen bonds with the $-\mathrm{OH}$ and $\mathrm{COOH}$ groups of AQOAT respectively. Such interactions of IND have previously reported with saccharin [29], polyvinyl pyrrolidone [30] and hydroxypropyl- $\beta$-cyclodextrin [31]. The DSC and XRD analysis showed the presence of IND in amorphous form within the extruded filaments and printed structured. Therefore, amorphous IND was prepared in order to identify the related peak shifting compared to the crystalline form. Fig. 6 demonstrates that crystalline of $\gamma$-form indomethacin contains benzoyl and acid carbonyl groups corresponding at $1717 \mathrm{~cm}$ ${ }^{1}$ and $1690 \mathrm{~cm}^{-1}$ respectively, whereas in amorphous form these peaks have moved towards lower wavenumbers at $1708 \mathrm{~cm}^{-1}$ (presence cyclic dimers) and $1680 \mathrm{~cm}^{-1}$ respectively. This observation is in good agreement with the published work by Taylor and Zografi [32]. In the $3 \mathrm{D}$ printed structure and filament the acid carbonyl group has moved to $1685 \mathrm{~cm}^{-1}$ whereas the benzoyl peak overlapped by the strong carbonyl peak of HPMCAS indicating H-bonding interactions. The amorphous state of IND in HPMC solid dispersions has also confirmed by other groups $[33,34]$, and it is attributed to the molecular mobility of IND within the polymer matrix. Another interesting observation is that the carbonyl peak of HPMCAS has shifted towards lower wavenumbers from $1745 \mathrm{~cm}^{-1}$ to $1733 \mathrm{~cm}^{-1}$ only for the $3 \mathrm{D}$ printed structure. This is due to the additional heat processing of the filament, suggesting stronger H-bonding interactions of the IND/HPMCAS blend.

\section{FT-IR Spectroscopy}




\section{Raman Mapping}

Fig. 7 shows the Raman spectra of the bulk compounds, extruded filament and 3D printed structures in the range of $1580 \mathrm{~cm}^{-1}-1700 \mathrm{~cm}^{-1}$ wavenumbers. Characteristic peaks of IND appear in the $1580-1625 \mathrm{~cm}^{-1}$ region, HPMCAS at $1740 \mathrm{~cm}^{-1}$ and PEG at $1200-$ $1300 \mathrm{~cm}^{-1}$. Previous studies showed that the peak at $1702 \mathrm{~cm}^{-1}$ corresponds to benzoyl carbonyl vibration while the peaks associated with the symmetrical stretching vibrations of the cyclic dimer formed by the carboxyl groups are not active in the Raman spectra [32]. In amorphous IND the peak is shifted towards lower wavenumbers at $1684 \mathrm{~cm}^{-1}$.

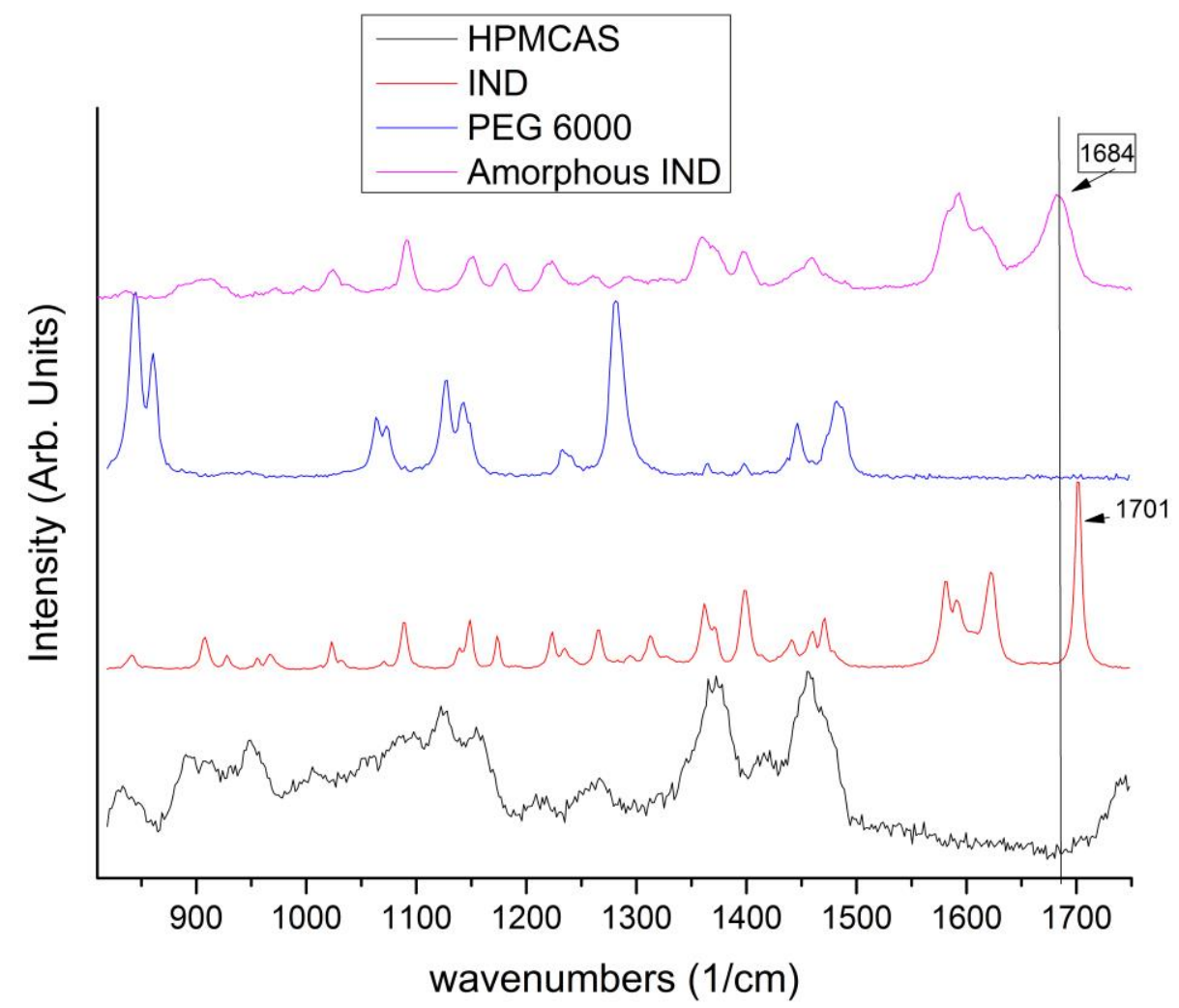

\section{Fig. 7: Raman Spectra of bulk HPMCAS, PEG 6000, crystalline and amorphous IND}

In order to investigate the IND homogeneity within the printed structures, multivariate data analysis PCA was applied to reduce the number of variables and identify the number of loadings, which consist the hyperspectral Raman data. Based on the eigenvalues, the hyperspectral data can be described by two principal components (PCs). This is a typical observation in multivariate data analysis of Raman spectra, which leads arbitrarily to the assumption that there are only two components in the system, as the first PC corresponds to the features of the strongest spectral contributors. It can be clearly observed from Fig. $8 \mathrm{~b}$ that the peaks of the first PC correspond to characteristic of amorphous IND and HPMCAS in the formulation, which overwhelms the PEG's peaks. For instance, the characteristic pattern of IND peaks appears in the $1550 \mathrm{~cm}^{-1}-1650 \mathrm{~cm}^{-1}$ region whereas several characteristic peaks of HPMCAS appear at $1366 \mathrm{~cm}^{-1}, 1456 \mathrm{~cm}^{-1}$, and $1739 \mathrm{~cm}^{-1}$ wavenumber. The characteristic peaks of PEG appear in the $4^{\text {th }} \mathrm{PC}$ at $840 \mathrm{~cm}^{-1}-885 \mathrm{~cm}^{-1}$ region and further at $1236 \mathrm{~cm}^{-1}$, $1282 \mathrm{~cm}^{-1}$ and at $1486 \mathrm{~cm}^{-1}$ wavenumbers, whereas the intermediate PCs are attributed to the noise and are not represented. Furthermore, the peaks, of PC4 assigned to IND and HPMCAS 
a)

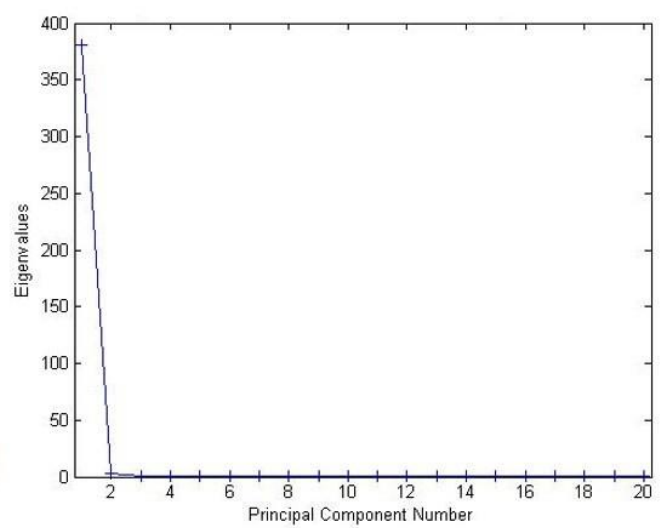

\section{b)}

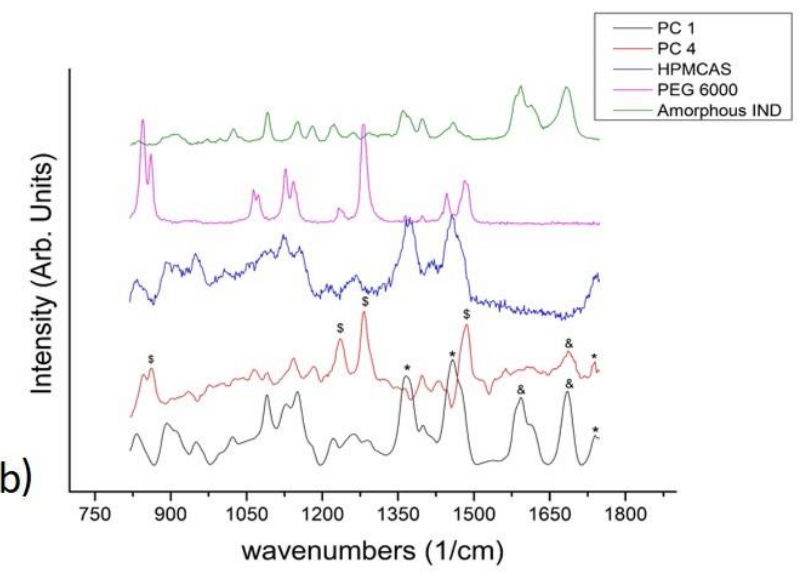

c)

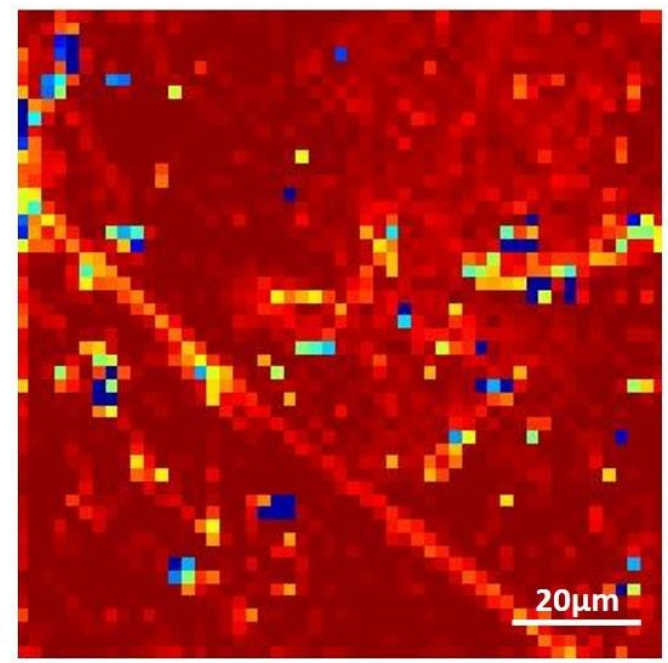

Fig. 8: a) Variation of Eigenvalues with the principal components, b) Comparison of HPMCAS and amorphous IND with the PC1 and PC4 where \$ correspond to PEG 6000, * to $H M P C A S$ and \& to IND, c) Concentration map of PCl, d) Concentration map of PC4

\section{Taste masking evaluation}

Taste masking efficiency of the 3D Starmix ${ }^{\circledR}$ designs was of the utmost importance for the purpose of paediatric applications $[35,36]$. We anticipated that the selection of the excipients and the HME processing will result in efficient masking of IND bitterness and increase the palatability of the printed designs. As shown in Fig. 9 the panellist evaluation revealed moderate bitterness for IND while slight and threshold for PEG and HPMCAS respectively. All 3D printed designs showed excellent taste masking with no bitterness at all and no aftertaste was reported. The masking effect is attributed to the drug - polymer interactions through $\mathrm{H}$ - bonding facilitated by HME processing [37]. The effective taste 
masking of the Starmix ${ }^{\circledR}$ designs suggests that 3D printed dosage forms can be successfully used in paediatric applications providing enhanced palatability.

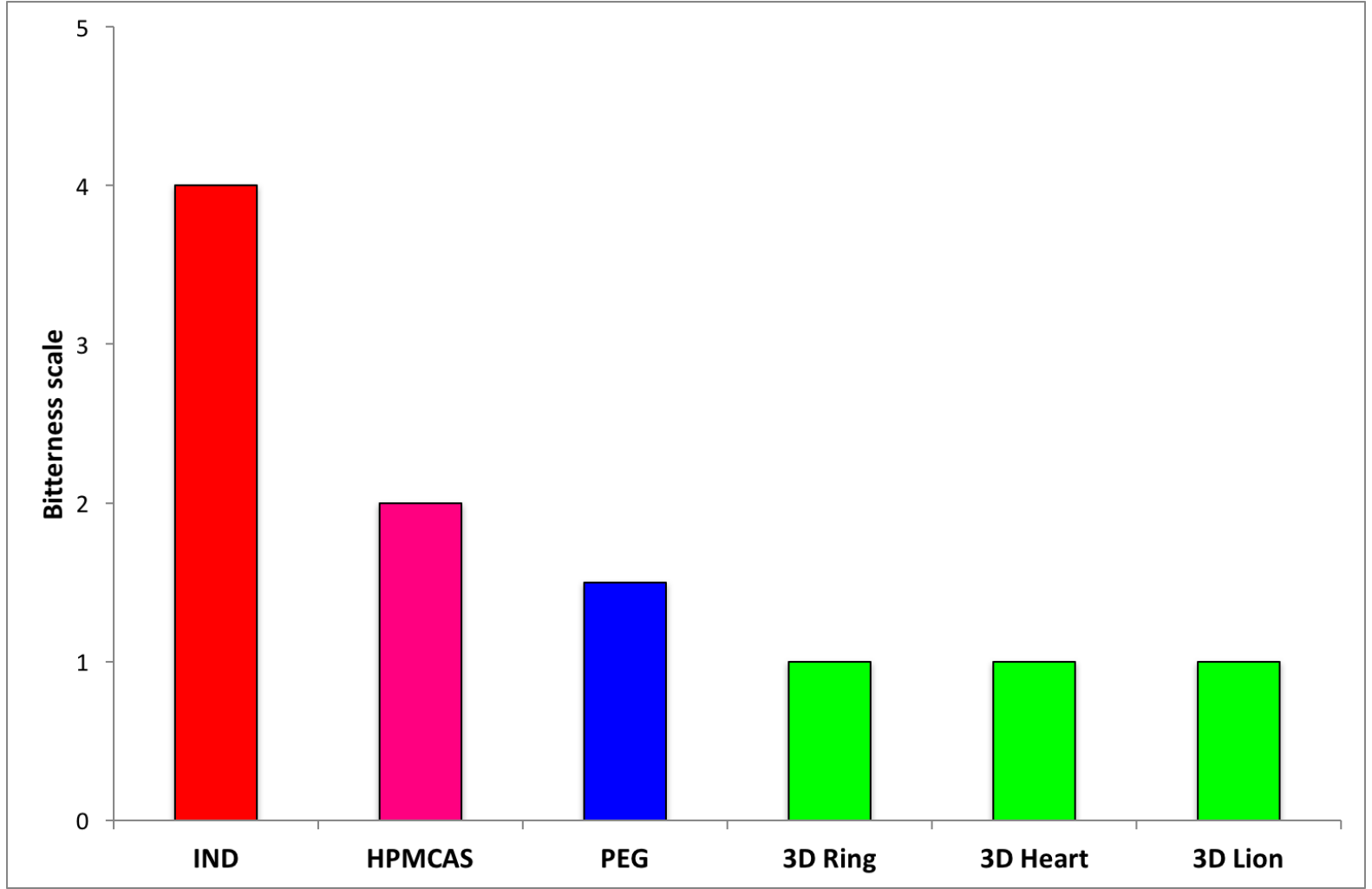

Fig. 9: Taste masking evaluation graph of bulk IND, HPMCAS, PEG and Starmix $®$ designs

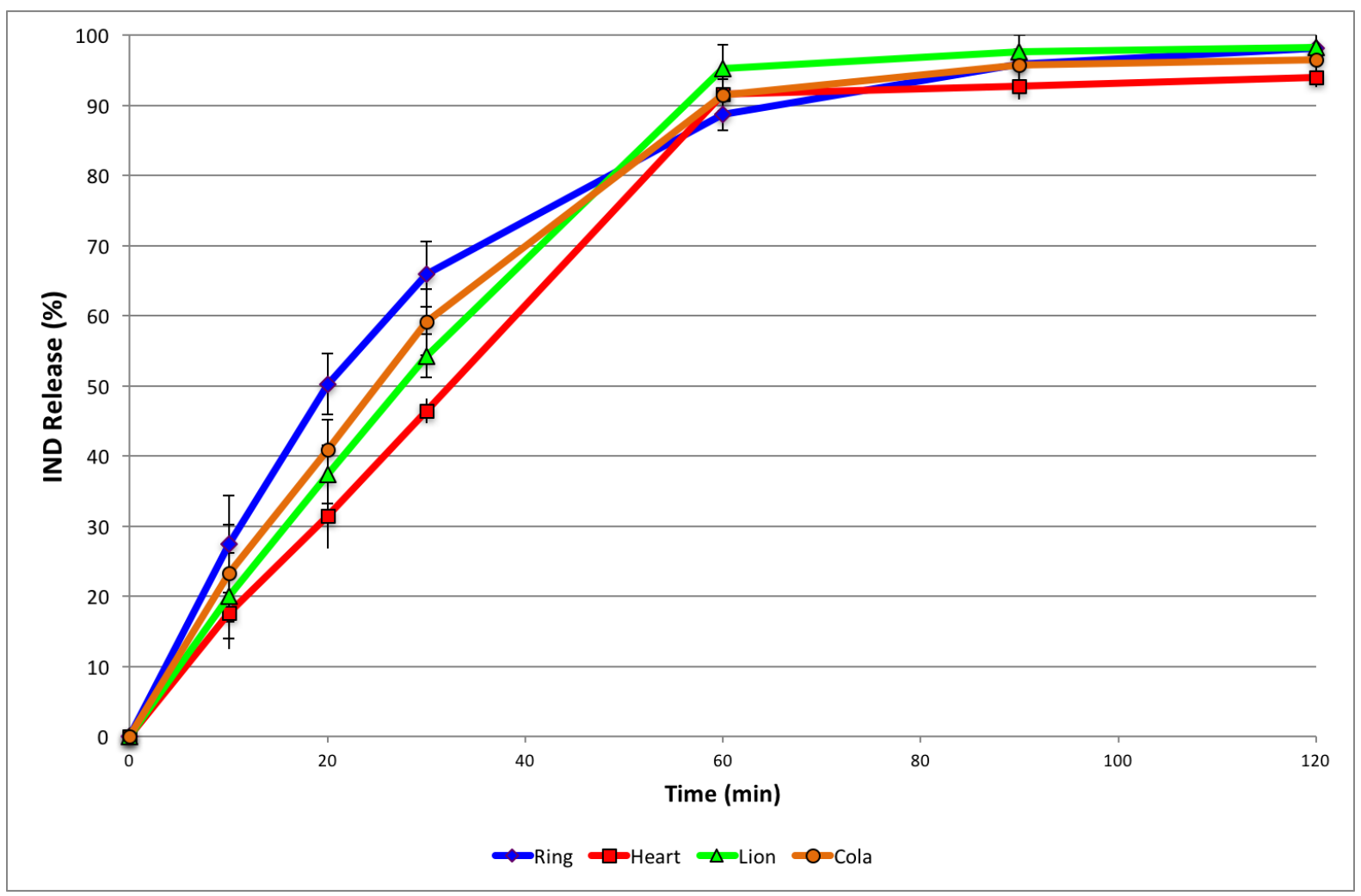

Figure 10: Dissolution rate of IND in three different $3 D$ printed structure 


\section{Dissolution studies}

As HPMCAS dissolves at $\mathrm{pH}>6$ we anticipated IND release in the mouth. However, children are expected to chew the tablets for a few minutes (up to $2 \mathrm{~min}$ ) and subsequently swallow them. For this reason, IND release was initially investigated similarly to Rachid et al by using small volumes ( $2 \mathrm{ml}$ ) of simulated saliva (data not shown) [38]. The release of IND was found to vary from $8.0-12 \times 10^{-3} \%$ which is negligible to cause any bitterness. These findings support the taste evaluation where the $3 \mathrm{D}$ printed tablets were found to mask IND bitternes.

In addition, the IND release profiles of four printed Starmix $®$ designs were conducted in phosphate buffer ( $\mathrm{pH}$ 7.4). The release profiles are illustrated in Fig 10 where it can be observed that for each printed form more than $80 \%$ of the drug was released within the first 60 min while the Starmix $®$ designs were completely dissolved.

The rapid IND release was facilitated by the fact that HPMCAS is highly soluble in $\mathrm{pH}>6.0$ and IND is molecularly dispersed within the printed forms. Interestingly, the release profiles of all Stramix $®$ designs are very similar and no significant difference was observed $(\mathrm{p}<0.05)$. It is also important to mention that the optimized printing process achieved release patterns independent of the printed designs. This is a crucial feature for the development of oral dosage forms with multiple designs or patterns. However, the alignment of drug release from 3D printed dosage forms was also observed by Goyanes et al. where tablets of different shapes and similar weights provided similar drug release profiles [20].

\section{CONCLUSION}

Starmix ${ }^{\circledR}$ designs were manufactured by using FDM 3D printing coupled with HME processing for the development of palatable paediatric dosage forms. FDM process was proved efficient in printing detailed Starmix ${ }^{\circledR}$ designs with high reproducibility, accuracy and content uniformity. Physicochemical characterisation studies showed that IND was molecularly dispersed in the HPMCAS matrix. The panellist taste evaluation showed excellent taste masking efficiency and hence enhanced palatability. The drug release profiles showed rapid IND release independent of the shape of printed design. Overall, we demonstrated that $3 \mathrm{D}$ printing can be used as an alternative manufacturing route for paediatric medicines.

\section{REFERECNES}

1. Strickley RG, Iwata Q, Wu S, Dahl TC. Pediatric drugs - a review of commercially available oral formulations, Journal of pharmaceutical sciences, 2008, 97:1731-1774.

2. Formulations of choice for the paediatric population. European Medicines Agency, (2006)

3. Lopez FL, Ernest TB, Tuleu C, Gul MO. Formulation approaches to pediatric oral drug delivery: benefits and limitations of current platforms, Expert opinion on drug delivery, 2015, 12:1727-1740.

4. Benavides S, Huynh D, Morgan J, Briars L. Approach to the pediatric prescription in a community pharmacy J Pediatr Pharmacol Ther. 2011, 16 (4):298-307.

5. Yin HS, Mendelsohn AL, Wolf MS, Parker RM, Fierman A, van Schaick L, Bazan IS, Kline MD, Dreyer BP. Parents' medication administration errors: role of dosing 
instruments and health literacy, Archives of pediatrics \& adolescent medicine, 2010, 164:181-186.

6. Mennella JA, Roberts KM, Mathew PS, Reed DR. Children's perceptions about medicines: individual differences and taste, BMC Pediatrics, 2015, 15:130.

7. Turner MA, Catapano M, Hirschfeld S, Giaquinto C Paediatric drug development: The impact of evolving regulations, Adv. Drug Deliv. Rev. 2014, 73:2-13.

8. Alhnan MA, Okwuosa TC, Sadia M, Wan KW, Ahmed W, Arafat B. Emergence of 3D Printed Dosage Forms: Opportunities and Challenges. Pharm Res. 2016 33(8):1817-32

9. Norman J, Madurawe RD, Moore CM, Khan MA, Khairuzzaman A. A new chapter in pharmaceutical manufacturing: 3D-printed drug products. Adv Drug Deliv Rev. 2017, 108:39-50

10. Sandler N, Preis M. Printed Drug-Delivery Systems for Improved Patient Treatment. Trends Pharmacol Sci. 2016, 37(12):1070-1080

11. Preis M, Öblom H. 3D-Printed Drugs for Children-Are We Ready Yet? AAPS PharmSciTech. 2017, 18(2):303-308

12. Sachs E, Cima M, Cornie J. Three-dimensional printing: rapid tooling and prototypes directly from a CAD model, CIRP Ann Manuf, Techn. 1990, 39: 201-204.

13. Khaled SA, Burley JC, Alexander MR, Roberts CR. Desktop 3D printing of controlled release pharmaceutical bilayer tablets, Int. J. Pharm. 2014,461:105-111.

14. Goyanes A, Chang H, Sedough D, Hatton GB, Wang J, Buanz A, Gaisford S, Basit AW. Fabrication of controlled-release budesonide tablets via desktop (FDM) 3D printing, Int. J. Pharm. 2015, 496:414-420.

15. Water JJ, Bohr A, Boetker J, Aho J, Sandler N, Nielsen HM, Rantanen J. ThreeDimensional Printing of Drug-Eluting Implants: Preparation of an Antimicrobial Polylactide Feedstock Material. J. Pharm. Sci. 2015, 104:1099-1107.

16. Khaled SA, Burley JC, Alexander MR, Yang J, Roberts CJ. 3D printing of five-in-one dose combination polypill with defined immediate and sustained release profiles. J. Control. Rel. 2015, 217:308-314.

17. Goyanes A, Det-Amornrat U, Wang J, Basit AW, Gaisford S. 3D scanning and 3D printing as innovative technologies for fabricating personalized topical drug delivery systems. J. Control. Rel., 2016, 234:41-48.

18. Busch SF, Weidenbach M, Fey M, Schäfer F, Probst T, Koch M. Optical Properties of 3D Printable Plastics in the THz Regime and their Application for 3D Printed $\mathrm{THz}$ Optics. J Infrared Millim Terahertz Waves. 2014,35:993-997.

19. Goyanes A, Buanz A, Hatton GB, Gaisford S, Basit AW. 3D printing of modifiedrelease aminosalicylate (4-ASA and 5-ASA) tablets. Eur. J. Pharm. Biopharm. 2015, 89:157-162.

20. Goyanes A, Martinez PR, Buanz A, Basit AW, Gaisford S. Effect of geometry on drug release from 3D printed tablets. Int. J. Pharma. 2015, 494: 657-663.

21. Skowyra J, Pietrzak K, Alhnan MA Fabrication of extended-release patient-tailored prednisolone tablets via fused deposition modelling (FDM) 3D printing. Eur. J. Pharm. Sci. 2015, 68:11-17.

22. Okwuosa TC, Stefaniak, D Arafat B, Isreb A, Wan K-W, Alhnan MA. A Lower Temperature FDM 3D Printing for the Manufacture of Patient-Specific Immediate Release Tablets. Pharm Res. 2016, 33(11):2704-12

23. Maniruzzaman M, Boateng JB, Bonnefille M, Aranyos A, Mitchell JC, Douroumis D. Taste masking of paracetamol by hot-melt extrusion: An in vitro and in vivo evaluation. Eur. J. Pharm. Biopharm. 2012, 80: 433-442. 
24. Goyanes A, Buanz AB, Basit AW, Gaisford S. Fused-filament 3D printing (3DP) for fabrication of tablets. Int. J. Pharma. 2014, 476: 88-92.

25. Genina N, Holländer J, Jukarainen H, Mäkilä E, Salonen J, Sandler N. Ethylene vinyl acetate (EVA) as a new drug carrier for 3D printed medical drug delivery devices. Eur. J. Pharm. Sci. 2015, 30(90):53-63

26. Melocchi A, Parietti F, Maroni A, Foppoli A, Gazzaniga A, Zema L. Hot-melt extruded filaments based on pharmaceutical grade polymers for 3D printing by fused deposition modelling. Int. J. Pharm. 2016, 509: 255-263.

27. Boetker J, Water JJ, Aho J, Arnfast L, Bohr A, Rantanen J. Modifying release characteristics from 3D printed drug-eluting products. Eur. J. Pharm. Sci. 2016, 90: 47-52.

28. El-Badry M, Fetih G, Fathy M. Improvement of solubility and dissolution rate of indomethacin by solid dispersions in Gelucire 50/13 and PEG4000, Saudi Pharm. J. 2009, 17: 217-225.

29. Zhang G-C, Lin H-L, Lin S-Y. Thermal analysis and FTIR spectral curve-fitting investigation of formation mechanism and stability of indomethacin-saccharin cocrystals via solid-state grinding process. J. Pharm. Biomed. Anal. 2012, 66:162169.

30. Lim RTY, Ng WK, Tan RB. Dissolution enhancement of indomethacin via amorphization using co-milling and supercritical co-precipitation processing, Powder Tech. 2013, 240: 79-87.

31. Bandi N, Wei W, Roberts CB, Kotra LP, Kompella UB. Preparation of budesonideand indomethacin-hydroxypropyl- $\beta$-cyclodextrin (HPBCD) complexes using a single-step, organic-solvent-free supercritical fluid process. Eur. J. Pharm. Sci. 2004, 23:159-168.

32. Taylor LS, Zografi G Spectroscopic Characterization of Interactions Between PVP and Indomethacin in Amorphous Molecular Dispersions. Pharm. Res. 1997, 14:16911698.

33. Ewing AV, Clarke GS, Kazarian SG. Stability of indomethacin with relevance to the release from amorphous solid dispersions studied with ATR-FTIR spectroscopic imaging. Eur. J. Pharm. Sci. 2014, 60: 64-71.

34. Chauhan H, Kuldipkumar A, Barder T, Medek A, Gu C-H, Atef E. Correlation of Inhibitory Effects of Polymers on Indomethacin Precipitation in Solution and Amorphous Solid Crystallization Based on Molecular Interaction. Pharm. Res. 2014, 31:500-515.

35. Walsh J, Cram A, Woertz K, Breitkreutz J, Winzenburg G, Turner R, Tuleu C, E.F. Initiative, Playing hide and seek with poorly tasting paediatric medicines: do not forget the excipients, Adv. Drug Deliv. Rev. 2014, 73:14-33.

36. Maniruzzaman M, Boateng JS, Chowdhry BZ, Snowden MJ, Douroumis D. A review on the taste masking of bitter APIs: hot-melt extrusion (HME) evaluation. Drug Dev. Ind. Pharm. 2014, 40:145-156.

37. Gryczke A, Schminke S, Maniruzzaman, Beck MJ, Douroumis D. Development and evaluation of orally disintegrating tablets (ODTs) containing Ibuprofen granules prepared by hot melt extrusion. Colloids Surf. B: Biointerfaces. 2011, 86: 275-284.

38. Rachid Q, Rawas-Qalaji M, Estelle F, Simons R, Simons KJ. Dissolution Testing of Sublingual Tablets: A Novel In Vitro Method. AAPS PharmSciTech. 2011, 12(2): 544-552. 